\title{
PERIODIC ORBITS OF THE FOURTH-ORDER NON-AUTONOMOUS DIFFERENTIAL EQUATION
} $u^{\prime \prime \prime \prime}+q u^{\prime \prime}+p u=\varepsilon F\left(t, u, u^{\prime}, u^{\prime \prime}, u^{\prime \prime \prime}\right)$

\author{
JAUME LLIBRE $^{1}$ AND AMAR MAKHLOUF ${ }^{2}$
}

\begin{abstract}
We provide sufficient conditions for the existence of periodic solutions of the fourth-order differential equation

$$
u^{\prime \prime \prime \prime}+q u^{\prime \prime}+p u=\varepsilon F\left(t, u, u^{\prime}, u^{\prime \prime}, u^{\prime \prime \prime}\right),
$$

where $q, p$ and $\varepsilon$ are real parameters, $\varepsilon$ is small and $F$ is a nonlinear nonautonomous periodic function with respect to $t$. Moreover we provide some applications.
\end{abstract}

\section{Introduction AND StATEMEnt of the MAin RESUlts}

The goal of this paper is to study the periodic solutions of the fourth-order non-autonomous differential equation

$$
u^{\prime \prime \prime \prime}+q u^{\prime \prime}+p u=\varepsilon F\left(t, u, u^{\prime}, u^{\prime \prime}, u^{\prime \prime \prime}\right),
$$

where $q, p$ and $\varepsilon$ are real parameters, $\varepsilon$ is small and $F$ is a nonlinear function. The prime denotes derivative with respect to an independent variable $t$.

In general to obtain analytically periodic solutions of a differential system is a very difficult task, usually impossible. Here using the averaging theory we reduce this difficult problem for the differential equations (1) to find the zeros of a nonlinear system of two or four equations. We must say that the averaging theory for finding periodic solutions in general does not provide all the periodic solutions of the system. For more information and details about the averaging theory see section 2 and the references quoted there.

Equations (1) appear in many places. For instance, Champneys [5] analyzes a class of equations (1) looking mainly for homoclinic orbits.

When $F= \pm u^{3}$, the equation (1) is called the Extended Fischer-Kolmogorov equation or the Swift-Hohenberg equation see [3,7], and in other places, see for instance the book [14] and [1, 4].

Some results on the periodic orbits for extended Fisher-Kolmogorov and SwiftHohenberg equations of the form

$$
u^{\prime \prime \prime \prime}+q u^{\prime \prime}+\alpha(t) u=f\left(t, u, u^{\prime}, u^{\prime \prime}\right),
$$

with $\alpha$ and $f$ functions, has been studied in [4] and in the references quoted there.

The differential equation (1) when $\mathrm{F}$ does not depend of $\mathrm{t}$ with $p<0$ has been studied in [11] taking $p=-1$ after a rescaling, and with $p>0$ it has been studied in $[8]$.

2000 Mathematics Subject Classification. 37G15, 37C80, 37C30.

Key words and phrases. periodic orbit, fourth-order differential equation, averaging theory. 
Our main result on the periodic solutions of the fourth-order non-autonomous differential equation (1) with $p<0$ is the following one.

Theorem 1. Assume that $p<0$ in the differential equation (1). Let

$$
\begin{aligned}
& \mathcal{F}_{1}\left(X_{0}, Y_{0}\right)=\frac{\alpha}{2 \pi} \int_{0}^{\frac{2 \pi}{\alpha}} \cos (\alpha t) F(t, A(t), B(t), C(t), D(t)) d t, \text { and } \\
& \mathcal{F}_{2}\left(X_{0}, Y_{0}\right)=-\frac{\alpha}{2 \pi} \int_{0}^{\frac{2 \pi}{\alpha}} \sin (\alpha t) F(t, A(t), B(t), C(t), D(t)) d t,
\end{aligned}
$$

be with $\sqrt{\frac{\sqrt{q^{2}-4 p}+q}{2}}=\alpha, \sqrt{\frac{\sqrt{q^{2}-4 p}-q}{2}}=\beta$, (or equivalently $p=-\alpha^{2} \beta^{2}<$ $\left.0, q=\alpha^{2}-\beta^{2}\right)$ with respectively, $\alpha$ and $\beta$, positive numbers, and

$$
\begin{aligned}
A(t) & =-\frac{Y_{0} \cos (\alpha t)+X_{0} \sin (\alpha t)}{\alpha\left(\alpha^{2}+\beta^{2}\right)} \\
B(t) & =\frac{-X_{0} \cos (\alpha t)+Y_{0} \sin (\alpha t)}{\alpha^{2}+\beta^{2}} \\
C(t) & =\frac{\alpha\left(Y_{0} \cos (\alpha t)+X_{0} \sin (\alpha t)\right)}{\alpha^{2}+\beta^{2}} \\
D(t) & =\frac{\alpha^{2}\left(X_{0} \cos (\alpha t)-Y_{0} \sin (\alpha t)\right)}{\alpha^{2}+\beta^{2}}
\end{aligned}
$$

If the function $F$ is $\frac{2 \pi}{\alpha}$-periodic with respect to the variable $t$, then for every $\left(X_{0}{ }^{*}, Y_{0}{ }^{*}\right)$ solution of the system

$$
\mathcal{F}_{k}\left(X_{0}, Y_{0}\right)=0, k=1,2,
$$

satisfying

$$
\operatorname{det}\left(\left.\frac{\partial\left(\mathcal{F}_{1}, \mathcal{F}_{2}\right)}{\partial\left(X_{0}, Y_{0}\right)}\right|_{\left(X_{0}, Y_{0}\right)=\left(X_{0}{ }^{*}, Y_{0}{ }^{*}\right)}\right) \neq 0
$$

the differential equation (1) has a periodic solution $u(t, \varepsilon)$ tending to the solution $u_{0}(t)$ given by

$$
-\frac{Y_{0}^{*} \cos (\alpha t)+X_{0}^{*} \sin (\alpha t)}{\alpha\left(\alpha^{2}+\beta^{2}\right)}
$$

of $u^{\prime \prime \prime \prime}+q u^{\prime \prime}+p u=0$ when $\varepsilon \rightarrow 0$. Note that this solution is periodic of period $\frac{2 \pi}{\alpha}$.

Theorem 1 is proved in section 3. Its proof is based in the averaging theory for computing periodic orbits, see section 2 .

Our main result on the periodic solutions of the fourth-order non-autonomous differential equation (1) with $p>0$ is the following one. 
Theorem 2. Assume that $p>0, q>0$ and $q^{2}-4 p>0$ in the differential equation (1). Let

$$
\begin{aligned}
& \mathcal{F}_{1}\left(X_{0}, Y_{0}, Z_{0}, V_{0}\right)=\frac{1}{2 \pi k} \int_{0}^{2 \pi k} \cos \left(\frac{m}{n} t\right) F(t, A(t), B(t), C(t), D(t)) d t, \\
& \mathcal{F}_{2}\left(X_{0}, Y_{0}, Z_{0}, V_{0}\right)=-\frac{1}{2 \pi k} \int_{0}^{2 \pi k} \sin \left(\frac{m}{n} t\right) F(t, A(t), B(t), C(t), D(t)) d t, \\
& \mathcal{F}_{3}\left(X_{0}, Y_{0}, Z_{0}, V_{0}\right)=\frac{1}{2 \pi k} \int_{0}^{2 \pi k} \cos \left(\frac{r}{s} t\right) F(t, A(t), B(t), C(t), D(t)) d t, \text { and } \\
& \mathcal{F}_{4}\left(X_{0}, Y_{0}, Z_{0}, V_{0}\right)=-\frac{1}{2 \pi k} \int_{0}^{2 \pi k} \sin \left(\frac{r}{s} t\right) F(t, A(t), B(t), C(t), D(t)) d t,
\end{aligned}
$$

be with $\sqrt{\frac{q+\sqrt{q^{2}-4 p}}{2}}=\frac{m}{n}, \sqrt{\frac{q-\sqrt{q^{2}-4 p}}{2}}=\frac{r}{s}$, (or equivalently $p=\frac{m^{2} r^{2}}{n^{2} s^{2}}, q=$ $\left.\frac{m^{2}}{n^{2}}+\frac{r^{2}}{s^{2}}\right)$ where $m, n, r$ and $s$ are positive integers, $(m, n)=(r, s)=1, k=$ l.c.m $(n, s)$ (least common multiple), and

$$
\begin{aligned}
& A(t)=K\left(\frac{m}{n}\left[V_{0} \cos \left(\frac{r}{s} t\right)+Z_{0} \sin \left(\frac{r}{s} t\right)\right]-\frac{r}{s}\left[Y_{0} \cos \left(\frac{m}{n} t\right)+X_{0} \sin \left(\frac{m}{n} t\right)\right]\right), \\
& B(t)=L\left(Z_{0} \cos \left(\frac{r}{s} t\right)-X_{0} \cos \left(\frac{m}{n} t\right)-V_{0} \sin \left(\frac{r}{s} t\right)+Y_{0} \sin \left(\frac{m}{n} t\right)\right), \\
& C(t)=L\left(-\frac{r}{s}\left[V_{0} \cos \left(\frac{r}{s} t\right)+Z_{0} \sin \left(\frac{r}{s} t\right)\right]+\frac{m}{n}\left[Y_{0} \cos \left(\frac{m}{n} t\right)+X_{0} \sin \left(\frac{m}{n} t\right)\right]\right), \\
& D(t)=L\left(-\frac{r^{2}}{s^{2}} Z_{0} \cos \left(\frac{r}{s} t\right)+\frac{m^{2}}{n^{2}} X_{0} \cos \left(\frac{m}{n} t\right)+\frac{r^{2}}{s^{2}} V_{0} \sin \left(\frac{r}{s} t\right)-\frac{m^{2}}{n^{2}} Y_{0} \sin \left(\frac{m}{n} t\right)\right),
\end{aligned}
$$

where

$$
K=\frac{n^{3} s^{3}}{m r\left(m^{2} s^{2}-n^{2} r^{2}\right)}, \quad L=\frac{n^{2} s^{2}}{m^{2} s^{2}-n^{2} r^{2}} .
$$

If the function $F$ is $2 \pi k$-periodic with respect to the variable $t$, then for every $\left(X_{0}{ }^{*}, Y_{0}{ }^{*}, Z_{0}{ }^{*}, V_{0}{ }^{*}\right)$ solution of the system

$$
\mathcal{F}_{k}\left(X_{0}, Y_{0}, Z_{0}, V_{0}\right)=0, k=1, \ldots, 4,
$$

satisfying

$$
\operatorname{det}\left(\left.\frac{\partial\left(\mathcal{F}_{1}, \mathcal{F}_{2}, \mathcal{F}_{3}, \mathcal{F}_{4}\right)}{\partial\left(X_{0}, Y_{0}, Z_{0}, V_{0}\right)}\right|_{\left(X_{0}, Y_{0}, Z_{0}, V_{0}\right)=\left(X_{0}{ }^{*}, Y_{0}{ }^{*}, Z_{0}{ }^{*}, V_{0}{ }^{*}\right)}\right) \neq 0,
$$

the differential equation (1) has a periodic solution $u(t, \varepsilon)$ tending to the solution $u_{0}(t)$ given by

$$
K\left[\frac{m}{n}\left[V_{0}^{*} \cos \left(\frac{r}{s} t\right)+Z_{0}^{*} \sin \left(\frac{r}{s} t\right)\right]-\frac{r}{s}\left[Y_{0}^{*} \cos \left(\frac{m}{n} t\right)+X_{0}^{*} \sin \left(\frac{m}{n} t\right)\right]\right]
$$

of $u^{\prime \prime \prime \prime}+q u^{\prime \prime}+p u=0$ when $\varepsilon \rightarrow 0$. Note that this solution is periodic of period $2 \pi k$.

Theorem 2 is proved in section 4. Its proof is done using a different averaging result than in the proof of Theorem 1. For other applications of the averaging theory see for instance [9] and [?].

Two applications of Theorem 1 and one application of Theorem 2 for studying the periodic solutions of equation (1) are given in the following three corollaries. They are proved in section 5 . 
Corollary 3. If $F\left(t, u, u^{\prime}, u^{\prime \prime}, u^{\prime \prime \prime}\right)=\sin t+u u^{\prime}-u^{\prime}+\left(u^{\prime}\right)^{3}$, then the differential equation (1) with $p=-1$ and $q=0$ has one periodic solution $u_{1}(t, \varepsilon)$ tending to the periodic solution $u_{1}(t)$ given by

$$
u_{1}(t)=-\frac{1}{3}\left(\frac{2 \cdot 2^{2 / 3}}{\sqrt[3]{9-\sqrt{65}}}+\sqrt[3]{2(9-\sqrt{65})}\right) \cos t
$$

of $u^{\prime \prime \prime \prime}-u=0$ when $\varepsilon \longrightarrow 0$.

Corollary 4. If $F\left(t, u, u^{\prime}, u^{\prime \prime}, u^{\prime \prime \prime}\right)=\left(1-u^{2}\right) \sin 2 t$, then the differential equation (1) with $p=-4$ and $q=3$ has four periodic solutions $u_{k}(t, \varepsilon)$ for $k=1,2,3,4$, tending to the periodic solutions

$$
\begin{aligned}
& u_{1}(t)=2 \cos (2 t), \quad u_{2}(t)=-2 \cos (2 t), \\
& u_{3}(t)=\frac{2}{\sqrt{3}} \sin (2 t), \quad u_{4}(t)=-\frac{2}{\sqrt{3}} \sin (2 t),
\end{aligned}
$$

of $u^{\prime \prime \prime \prime}+3 u^{\prime \prime}-4 u=0$ when $\varepsilon \longrightarrow 0$.

Corollary 5. If $F\left(t, u, u^{\prime}, u^{\prime \prime}, u^{\prime \prime \prime}\right)=(1-u) \sin t$, then the differential equation (1) with $p=\frac{1}{36}$ and $q=\frac{13}{36}$ has one periodic solution $u_{1}(t, \varepsilon)$, tending to the periodic solution

$$
u_{1}(t)=\frac{36}{5}\left(3 V_{0}^{*} \cos \left(\frac{t}{3}\right)+3 Z_{0}^{*} \sin \left(\frac{t}{3}\right)-2 X_{0}^{*} \sin \left(\frac{t}{2}\right)\right),
$$

of $u^{\prime \prime \prime \prime}+\frac{1}{36} u^{\prime \prime}+\frac{13}{36} u=0$ when $\varepsilon \longrightarrow 0$, where

$$
\begin{aligned}
X_{0}^{*} & =-\frac{9625}{108(52992 \sqrt{3}-29645 \pi)}, \\
Z_{0}^{*} & =\frac{1925\left(-17253286281216+9678017986560 \sqrt{3} \pi-5428725209600 \pi^{2}+338348019625 \sqrt{3} \pi^{3}\right)}{864(52992 \sqrt{3}-29645 \pi)\left(-8424456192+3141895680 \sqrt{3} \pi-878826025 \pi^{2}\right)}, \\
V_{0}^{*} & =\frac{1925\left(-325582848+121918720 \sqrt{3} \pi-34239975 \pi^{2}\right)}{2592\left(-8424456192+3141895680 \sqrt{3} \pi-878826025 \pi^{2}\right)} .
\end{aligned}
$$

\section{BASIC RESUlts ON AVERAGING THEORY}

In this section we present the basic results from the averaging theory that we shall need for proving the main results of this paper.

We consider the problem of the bifurcation of $T$-periodic solutions from differential systems of the form

$$
\dot{\mathbf{x}}=F_{0}(t, \mathbf{x})+\varepsilon F_{1}(t, \mathbf{x})+\varepsilon^{2} F_{2}(t, \mathbf{x}, \varepsilon),
$$

with $\varepsilon=0$ to $\varepsilon \neq 0$ sufficiently small. Here the functions $F_{0}, F_{1}: \mathbb{R} \times \Omega \rightarrow \mathbb{R}^{n}$ and $F_{2}: \mathbb{R} \times \Omega \times\left(-\varepsilon_{0}, \varepsilon_{0}\right) \rightarrow \mathbb{R}^{n}$ are $\mathcal{C}^{2}$ functions, $T$-periodic in the first variable, and $\Omega$ is an open subset of $\mathbb{R}^{n}$. The main assumption is that the unperturbed system

$$
\dot{\mathbf{x}}=F_{0}(t, \mathbf{x}),
$$

has a submanifold of periodic solutions. A solution of this problem is given using the averaging theory.

Let $\mathbf{x}(t, \mathbf{z}, \varepsilon)$ be the solution of the system (8) such that $\mathbf{x}(0, \mathbf{z}, \varepsilon)=\mathbf{z}$. We write the linearization of the unperturbed system along a periodic solution $\mathbf{x}(t, \mathbf{z}, 0)$ as

$$
\dot{\mathbf{y}}=D_{\mathbf{x}} F_{0}(t, \mathbf{x}(t, \mathbf{z}, 0)) \mathbf{y} \text {. }
$$


In what follows we denote by $M_{\mathbf{z}}(t)$ some fundamental matrix of the linear differential system $(9)$, and by $\xi: \mathbb{R}^{k} \times \mathbb{R}^{n-k} \rightarrow \mathbb{R}^{k}$ the projection of $\mathbb{R}^{n}$ onto its first $k$ coordinates; i.e. $\xi\left(x_{1}, \ldots, x_{n}\right)=\left(x_{1}, \ldots, x_{k}\right)$.

We assume that there exists a $k$-dimensional submanifold $\mathcal{Z}$ of $\Omega$ filled with $T$-periodic solutions of (8). Then an answer to the problem of bifurcation of $T$ periodic solutions from the periodic solutions contained in $\mathcal{Z}$ for system (7) is given in the following result.

Theorem 6. Let $W$ be an open and bounded subset of $\mathbb{R}^{k}$, and let $\beta: \mathrm{Cl}(W) \rightarrow$ $\mathbb{R}^{n-k}$ be a $\mathcal{C}^{2}$ function. We assume that

(i) $\mathcal{Z}=\left\{\mathbf{z}_{\alpha}=(\alpha, \beta(\alpha)), \alpha \in \mathrm{Cl}(W)\right\} \subset \Omega$ and that for each $\mathbf{z}_{\alpha} \in \mathcal{Z}$ the solution $\mathbf{x}\left(t, \mathbf{z}_{\alpha}\right)$ of $(8)$ is $T$-periodic;

(ii) for each $\mathbf{z}_{\alpha} \in \mathcal{Z}$ there is a fundamental matrix $M_{\mathbf{z}_{\alpha}}(t)$ of (9) such that the matrix $M_{\mathbf{z}_{\alpha}}^{-1}(0)-M_{\mathbf{z}_{\alpha}}^{-1}(T)$ has in the upper right corner the $k \times(n-k)$ zero matrix, and in the lower right corner a $(n-k) \times(n-k)$ matrix $\Delta_{\alpha}$ with $\operatorname{det}\left(\Delta_{\alpha}\right) \neq 0$.

We consider the function $\mathcal{F}: \mathrm{Cl}(W) \rightarrow \mathbb{R}^{k}$

$$
\mathcal{F}(\alpha)=\xi\left(\frac{1}{T} \int_{0}^{T} M_{\mathbf{z}_{\alpha}}^{-1}(t) F_{1}\left(t, \mathbf{x}\left(t, \mathbf{z}_{\alpha}\right)\right) d t\right) .
$$

If there exists $a \in W$ with $\mathcal{F}(a)=0$ and $\operatorname{det}((d \mathcal{F} / d \alpha)(a)) \neq 0$, then there is a $T$-periodic solution $\varphi(t, \varepsilon)$ of system $(7)$ such that $\varphi(0, \varepsilon) \rightarrow \mathbf{z}_{a}$ as $\varepsilon \rightarrow 0$.

Theorem 6 goes back to Malkin [13] and Roseau [15], for a shorter proof see [2].

We assume that there exists an open set $V$ with $\mathrm{Cl}(V) \subset \Omega$ such that for each $\mathbf{z} \in \mathrm{Cl}(V), \mathbf{x}(t, \mathbf{z}, 0)$ is $T$-periodic, where $\mathbf{x}(t, \mathbf{z}, 0)$ denotes the solution of the unperturbed system (8) with $\mathbf{x}(0, \mathbf{z}, 0)=\mathbf{z}$. The set $\mathrm{Cl}(V)$ is isochronous for the system (7); i.e. it is a set formed only by periodic orbits, all of them having the same period. Then, an answer to the problem of the bifurcation of $T$-periodic solutions from the periodic solutions $\mathbf{x}(t, \mathbf{z}, 0)$ contained in $\mathrm{Cl}(V)$ is given in the following result.

Theorem 7. [Perturbations of an isochronous set] We assume that there exists an open and bounded set $V$ with $\mathrm{Cl}(V) \subset \Omega$ such that for each $\mathbf{z} \in \mathrm{Cl}(V)$, the solution $\mathbf{x}(t, \mathbf{z})$ is T-periodic, then we consider the function $\mathcal{F}: \mathrm{Cl}(V) \rightarrow \mathbb{R}^{n}$

$$
\mathcal{F}(\mathbf{z})=\int_{0}^{T} M_{\mathbf{z}}^{-1}(t, \mathbf{z}) F_{1}(t, \mathbf{x}(t, \mathbf{z})) d t .
$$

If there exists $a \in V$ with $\mathcal{F}(a)=0$ and $\operatorname{det}((d \mathcal{F} / d \mathbf{z})(a)) \neq 0$, then there exists $a$ $T$-periodic solution $\varphi(t, \varepsilon)$ of system $(7)$ such that $\varphi(0, \varepsilon) \rightarrow a$ as $\varepsilon \rightarrow 0$.

For a proof of Theorem 7 see Corollary 1 of [2].

\section{Proof of Theorem 1}

Introducing the variables $(x, y, z, v)=\left(u, u^{\prime}, u^{\prime \prime}, u^{\prime \prime \prime}\right)$ we write the fourth-order differential equation (1) as a first-order differential system defined in an open subset 
$\Omega$ of $\mathbb{R}^{4}$. Thus we have the differential system

$$
\begin{aligned}
& x^{\prime}=y, \\
& y^{\prime}=z, \\
& z^{\prime}=v, \\
& v^{\prime}=-p x-q z+\varepsilon F(t, x, y, z, v) .
\end{aligned}
$$

Of course as before the dot denotes derivative with respect to the independent variable $t$. System (12) with $\varepsilon=0$ will be called the unperturbed system, otherwise we have the perturbed system. The unperturbed system has a unique singular point, the origin. Since $p<0$ the origin has one pair of imaginary eigenvalues and two real eigenvalues, more precisely the eigenvalues are

$$
\pm \sqrt{\frac{\sqrt{q^{2}-4 p}+q}{2}} i= \pm \alpha i, \quad \pm \sqrt{\frac{\sqrt{q^{2}-4 p}-q}{2}}= \pm \beta .
$$

We shall write system (12) in such a way that the linear part at the origin will be in its real Jordan normal form. Then, doing the change of variables $(x, y, z, v) \rightarrow$ $(X, Y, Z, V)$ given by

$$
\left(\begin{array}{l}
X \\
Y \\
Z \\
V
\end{array}\right)=\left(\begin{array}{cccc}
0 & -\beta^{2} & 0 & 1 \\
\frac{p}{\alpha} & 0 & \alpha & 0 \\
\frac{p}{\beta} & \alpha^{2} & -\beta & 1 \\
-\frac{p}{\beta} & \alpha^{2} & \beta & 1
\end{array}\right)\left(\begin{array}{l}
x \\
y \\
z \\
v
\end{array}\right)
$$

the differential system (12) becomes

$$
\begin{aligned}
& X^{\prime}=-\alpha Y+\varepsilon F(t, a(t), b(t), c(t), d(t)), \\
& Y^{\prime}=\alpha X \\
& Z^{\prime}=-\beta Z+\varepsilon F(t, a(t), b(t), c(t), d(t)), \\
& V^{\prime}=\beta V+\varepsilon F(t, a(t), b(t), c(t), d(t)),
\end{aligned}
$$

with

$$
\begin{aligned}
& \left.a(t)=\frac{-1}{4 \alpha \beta^{2}\left(\alpha^{2}+\beta^{2}\right)}\left[\left(\alpha^{2}-\beta^{2}\right)(-2 Y-V+Z)+\left(\alpha^{2}+\beta^{2}\right)\right)(2 Y-V+Z)\right], \\
& b(t)=\frac{1}{2\left(\alpha^{2}+\beta^{2}\right)}[V-2 X+Z], \\
& c(t)=\frac{1}{2\left(\alpha^{2}+\beta^{2}\right)}[2 \alpha Y+\beta(V-Z)], \\
& d(t)=\frac{1}{4\left(\alpha^{2}+\beta^{2}\right)}\left[\left(\beta^{2}-\alpha^{2}\right)(V-2 X+Z)+\left(\alpha^{2}+\beta^{2}\right)(V+2 X+Z)\right] .
\end{aligned}
$$

Note that the linear part of the differential system (13) at the origin is in its real normal form of Jordan. 
We shall apply Theorem 6 to the differential system (13). We note that system (13) can be written as system (7) taking

$$
\mathbf{x}=\left(\begin{array}{c}
X \\
Y \\
Z \\
V
\end{array}\right), \quad t=t, \quad F_{0}(t, \mathbf{x})=\left(\begin{array}{c}
-\alpha Y \\
\alpha X \\
-\beta Z \\
\beta V
\end{array}\right)
$$

and

$$
F_{1}(t, \mathbf{x})=\left(\begin{array}{c}
F(t, a(t), b(t), c(t), d(t)) \\
0 \\
F(t, a(t), b(t), c(t), d(t)) \\
F(t, a(t), b(t), c(t), d(t))
\end{array}\right)
$$

We shall study the periodic solutions of system (8) in our case, i.e. the periodic solutions of system (13) with $\varepsilon=0$. These periodic solutions are

$$
\left(\begin{array}{c}
X(t) \\
Y(t) \\
Z(t) \\
V(t)
\end{array}\right)=\left(\begin{array}{c}
X_{0} \cos (\alpha t)-Y_{0} \sin (\alpha t) \\
Y_{0} \cos (\alpha t)+X_{0} \sin (\alpha t) \\
0 \\
0
\end{array}\right)
$$

This set of periodic orbits has dimension two, all having the same period $\frac{2 \pi}{\alpha}$. To look for the periodic solutions of our equation (13) we must calculate the zeros $\mathbf{z}=\left(X_{0}, Y_{0}\right)$ of the system $\mathcal{F}(\mathbf{z})=0$, where $\mathcal{F}(\mathbf{z})$ is given by (10). The fundamental matrix $M(t)$ of the differential system (13) with $\varepsilon=0$ along any periodic solution is

$$
M(t)=M_{\mathbf{z}}(t)=\left(\begin{array}{cccc}
\cos (\alpha t) & -\sin (\alpha t) & 0 & 0 \\
\sin (\alpha t) & \cos (\alpha t) & 0 & 0 \\
0 & 0 & \exp (-\beta t) & 0 \\
0 & 0 & 0 & \exp (\beta t)
\end{array}\right)
$$

The inverse matrix of $M(t)$ is

$$
M I(t)=\left(\begin{array}{cccc}
\cos (\alpha t) & \sin (\alpha t) & 0 & 0 \\
-\sin (\alpha t) & \cos (\alpha t) & 0 & 0 \\
0 & 0 & \exp (\beta t) & 0 \\
0 & 0 & 0 & \exp (-\beta t)
\end{array}\right) .
$$


It verifies

$$
M I(0)-M I\left(\frac{2 \pi}{\alpha}\right)=\left(\begin{array}{cccc}
0 & 0 & 0 & 0 \\
0 & 0 & 0 & 0 \\
0 & 0 & 1-\exp \left(2 \pi \frac{\beta}{\alpha}\right) & 0 \\
0 & 0 & 0 & 1-\exp \left(-2 \pi \frac{\beta}{\alpha}\right)
\end{array}\right) .
$$

Consequently all the assumptions of Theorem 6 are satisfied. Therefore we must study the zeros in $\mathrm{W}$ of the system $\mathcal{F}(\mathbf{z})=0$ of two equations with two unknowns, where $\mathrm{W}$ and $\mathcal{F}$ are given in the statement of Theorem 6 . More precisely, we have $\mathcal{F}(\mathbf{z})=\left(\mathcal{F}_{1}\left(X_{0}, Y_{0}\right), \mathcal{F}_{2}\left(X_{0}, Y_{0}\right)\right)$ where

$$
\begin{aligned}
& \mathcal{F}_{1}\left(X_{0}, Y_{0}\right)=\frac{\alpha}{2 \pi} \int_{0}^{\frac{2 \pi}{\alpha}} \cos (\alpha t) F(t, A(t), B(t), C(t), D(t)) d t, \\
& \mathcal{F}_{2}\left(X_{0}, Y_{0}\right)=-\frac{\alpha}{2 \pi} \int_{0}^{\frac{2 \pi}{\alpha}} \sin (\alpha t) F(t, A(t), B(t), C(t), D(t)) d t,
\end{aligned}
$$

with $A(t), B(t), C(t)$ and $D(t)$ as in the statement of Theorem 1.

The zeros $\left(X_{0}^{*}, Y_{0}^{*}\right)$ of system

$$
\left(\begin{array}{l}
\mathcal{F}_{1}\left(X_{0}, Y_{0}\right) \\
\left.\mathcal{F}_{2}\left(X_{0}, Y_{0}\right)\right)
\end{array}\right)=\left(\begin{array}{l}
0 \\
0
\end{array}\right)
$$

with respect to the variables $X_{0}$ and $Y_{0}$ provide periodic orbits of system (13) with $\varepsilon \neq 0$ sufficiently small if they are simple, i.e. if

$$
\operatorname{det}\left(\left.\frac{\partial\left(\mathcal{F}_{1}, \mathcal{F}_{2}\right)}{\partial\left(X_{0}, Y_{0}\right)}\right|_{\left(X_{0}, Y_{0}\right)=\left(X_{0}^{*}, Y_{0}^{*}\right)}\right) \neq 0 .
$$

Going back through the change of variable, for every simple zero $\left(X_{0}^{*}, Y_{0}^{*}\right)$ of system (14), we obtain a $\frac{2 \pi}{\alpha}$ periodic solution $u(t)$ of the differential equation (1) for $\varepsilon \neq 0$ sufficiently small such that $u(t)$ tends to the periodic solution

$$
u_{0}(t)=-\frac{Y_{0}^{*} \cos (\alpha t)+X_{0}^{*} \sin (\alpha t)}{\alpha\left(\alpha^{2}+\beta^{2}\right)}
$$

of $u^{\prime \prime \prime \prime}+q u^{\prime \prime}+p u=0$ when $\varepsilon \rightarrow 0$. Note that this solution is periodic of period $\frac{2 \pi}{\alpha}$. This completes the proof of Theorem 1 .

\section{Proof of Theorem 2}

As in the proof of Theorem 1 the differential equation (1) can be written as the differential system (12). The unperturbed system has a unique singular point, the origin. Since $p>0$ and $q^{2}-4 p>0$ the origin has two pairs of imaginary eigenvalues

$$
\pm \sqrt{\frac{q+\sqrt{q^{2}-4 p}}{2}} i= \pm \frac{m}{n} i, \quad \pm \sqrt{\frac{q-\sqrt{q^{2}-4 p}}{2}} i= \pm \frac{r}{s} i
$$


We shall write system (12) in such a way that the linear part at the origin will be in its real Jordan normal form. Then, doing the change of variables $(x, y, z, v) \rightarrow$ $(X, Y, Z, V)$ given by

$$
\left(\begin{array}{l}
X \\
Y \\
Z \\
V
\end{array}\right)=\left(\begin{array}{cccc}
0 & \frac{r^{2}}{s^{2}} & 0 & 1 \\
\frac{p n}{m} & 0 & \frac{m}{n} & 0 \\
0 & \frac{m^{2}}{n^{2}} & 0 & 1 \\
\frac{p s}{r} & 0 & \frac{r}{s} & 0
\end{array}\right)\left(\begin{array}{l}
x \\
y \\
z \\
v
\end{array}\right)
$$

the differential system (12) becomes

$$
\begin{aligned}
X^{\prime} & =-\frac{m}{m} Y+\varepsilon F(t, a(t), b(t), c(t), d(t)), \\
Y^{\prime} & =\frac{m}{m} X, \\
Z^{\prime} & =-\frac{r}{s} V+\varepsilon F(t, a(t), b(t), c(t), d(t)) \\
V^{\prime} & =\frac{r}{s} Z,
\end{aligned}
$$

with

$$
\begin{aligned}
& a(t)=\frac{n^{3} s^{3}}{m r\left(m^{2} s^{2}-n^{2} r^{2}\right)}\left[\frac{m}{n} V-\frac{r}{s} Y\right], \\
& b(t)=\frac{n^{2} s^{2}}{m^{2} s^{2}-n^{2} r^{2}}[Z-X], \\
& c(t)=\frac{n^{2} s^{2}}{m^{2} s^{2}-n^{2} r^{2}}\left[\frac{m}{n} Y-\frac{r}{s} V\right], \\
& d(t)=\frac{n^{2} s^{2}}{m^{2} s^{2}-n^{2} r^{2}}\left[\frac{m^{2}}{n^{2}} X-\frac{r^{2}}{s^{2}} Z\right] .
\end{aligned}
$$

Note that the linear part of the differential system (16) at the origin is in its real Jordan normal form.

We shall apply Theorem 7 to the differential system (16). We note that system (16) can be written as system (7) taking

$$
\mathbf{x}=\left(\begin{array}{c}
X \\
Y \\
Z \\
V
\end{array}\right), \quad t=t, \quad F_{0}(t, \mathbf{x})=\left(\begin{array}{c}
-\frac{m}{n} Y \\
\frac{m}{n} X \\
-\frac{r}{s} V \\
\frac{r}{s} Z
\end{array}\right),
$$

and

$$
F_{1}(t,, \mathbf{x})=\left(\begin{array}{c}
F(t, a(t), b(t), c(t), d(t)) \\
0 \\
F(t, a(t), b(t), c(t), d(t)) \\
0
\end{array}\right)
$$


We shall study the periodic solutions of system (8) in our case, i.e. the periodic solutions of system (16) with $\varepsilon=0$. These periodic solutions are

$$
\left(\begin{array}{c}
X(t) \\
Y(t) \\
Z(t) \\
V(t)
\end{array}\right)=\left(\begin{array}{c}
X_{0} \cos \left(\frac{m}{n} t\right)-Y_{0} \sin \left(\frac{m}{n} t\right) \\
Y_{0} \cos \left(\frac{m}{n} t\right)+X_{0} \sin \left(\frac{m}{n} t\right) \\
Z_{0} \cos \left(\frac{r}{s} t\right)-V_{0} \sin \left(\frac{r}{s} t\right) \\
V_{0} \cos \left(\frac{r}{s} t\right)+Z_{0} \sin \left(\frac{r}{s} t\right)
\end{array}\right) .
$$

This set of periodic orbits has dimension four, all having the same period $2 \pi k$, where $\mathrm{k}$ is defined in the statement of Theorem 2. To look for the periodic solutions of our equation (1) we must calculate the zeros $\mathbf{z}=\left(X_{0}, Y_{0}, Z_{0}, V_{0}\right)$ of the system $\mathcal{F}(\mathbf{z})=0$, where $\mathcal{F}(\mathbf{z})$ is given by (11). The fundamental matrix $M(t)$ of the differential system (16) with $\varepsilon=0$ along any periodic solution is

$$
M(t)=M_{\mathbf{z}}(t)=\left(\begin{array}{cccc}
\cos \left(\frac{m}{n} t\right) & -\sin \left(\frac{m}{n} t\right) & 0 & 0 \\
\sin \left(\frac{m}{n} t\right) & \cos \left(\frac{m}{n} t\right) & 0 & 0 \\
0 & 0 & \cos \left(\frac{r}{s} t\right) & -\sin \left(\frac{r}{s}\right) \\
0 & 0 & \sin \left(\frac{r}{s} t\right) & \cos \left(\frac{r}{s}\right)
\end{array}\right) .
$$

Now computing the function $\mathcal{F}(\mathbf{z})$ given in (11) we got that the system $\mathcal{F}(\mathbf{z})=0$ can be written as

$$
\left(\begin{array}{c}
\mathcal{F}_{1}\left(X_{0}, Y_{0}, Z_{0}, V_{0}\right) \\
\mathcal{F}_{2}\left(X_{0}, Y_{0}, Z_{0}, V_{0}\right) \\
\mathcal{F}_{3}\left(X_{0}, Y_{0}, Z_{0}, V_{0}\right) \\
\mathcal{F}_{4}\left(X_{0}, Y_{0}, Z_{0}, V_{0}\right)
\end{array}\right)=\left(\begin{array}{l}
0 \\
0 \\
0 \\
0
\end{array}\right)
$$

where

$$
\begin{aligned}
& \mathcal{F}_{1}\left(X_{0}, Y_{0}, Z_{0}, V_{0}\right)=\frac{1}{2 \pi k} \int_{0}^{2 \pi k} \cos \left(\frac{m}{n} t\right) F(t, A(t), B(t), C(t), D(t)) d t \\
& \mathcal{F}_{2}\left(X_{0}, Y_{0}, Z_{0}, V_{0}\right)=-\frac{1}{2 \pi k} \int_{0}^{2 \pi k} \sin \left(\frac{m}{n} t\right) F(t, A(t), B(t), C(t), D(t)) d t \\
& \mathcal{F}_{3}\left(X_{0}, Y_{0}, Z_{0}, V_{0}\right)=\frac{1}{2 \pi k} \int_{0}^{2 \pi k} \cos \left(\frac{r}{s} t\right) F(t, A(t), B(t), C(t), D(t)) d t \\
& \mathcal{F}_{4}\left(X_{0}, Y_{0}, Z_{0}, V_{0}\right)=-\frac{1}{2 \pi k} \int_{0}^{2 \pi k} \sin \left(\frac{r}{s} t\right) F(t, A(t), B(t), C(t), D(t)) d t
\end{aligned}
$$

with $A(t), B(t), C(t)$ and $D(t)$ as in the statement of Theorem 2.

The zeros $\left(X_{0}^{*}, Y_{0}^{*}, Z_{0}^{*}, V_{0}^{*}\right)$ of system (4) with respect to the variables $X_{0}, Y_{0}$, $Z_{0}$ and $V_{0}$ provide periodic orbits of system (16) with $\varepsilon \neq 0$ sufficiently small if they are simple, i.e. if

$$
\operatorname{det}\left(\left.\frac{\partial\left(\mathcal{F}_{1}, \mathcal{F}_{2}, \mathcal{F}_{3}, \mathcal{F}_{4}\right)}{\partial\left(X_{0}, Y_{0}, Z_{0}, V_{0}\right)}\right|_{\left(X_{0}, Y_{0}, Z_{0}, V_{0}\right)=\left(X_{0^{*}}, Y_{0} *, Z_{0} *, V_{0}^{*}\right)}\right) \neq 0 .
$$


Going back through the change of variables, for every simple zero $\left(X_{0}^{*}, Y_{0}^{*}, Z_{0}^{*}, V_{0}^{*}\right)$ of system (4), we obtain a $2 \pi k$ periodic solution $u(t)$ of the differential equation (1) for $\varepsilon \neq 0$ sufficiently small such that $u(t)$ tends to the periodic solution

$$
K\left[\frac{m}{n}\left[V_{0}^{*} \cos \left(\frac{r}{s} t\right)+Z_{0}^{*} \sin \left(\frac{r}{s} t\right)\right]-\frac{r}{s}\left[Y_{0}^{*} \cos \left(\frac{m}{n} t\right)+X_{0}^{*} \sin \left(\frac{m}{n} t\right)\right]\right]
$$

of $u^{\prime \prime \prime \prime}+q u^{\prime \prime}+p u=0$ when $\varepsilon \rightarrow 0$, where $K$ is defined in the statement of

Theorem 2 . Note that this solution is periodic of period $2 \pi k$. This completes the proof of Theorem 2.

\section{Proof of Corollaries 3,4 and 5}

Proof of Corollary 3. We have the equation

$$
u^{\prime \prime \prime \prime}-u=\varepsilon\left(\sin t+u u^{\prime}-u^{\prime}+\left(u^{\prime}\right)^{3}\right),
$$

which corresponds to the case $p=-1, q=0$ and $F(t, x, y, z, v)=\sin (t)+x y-y+y^{3}$. We obtain $\alpha=\beta=1$. The functions $\mathcal{F}_{1}$ and $\mathcal{F}_{2}$ of Theorem 1 are

$$
\begin{aligned}
& \mathcal{F}_{1}\left(X_{0}, Y_{0}\right)=-\frac{1}{64} X_{0}\left[3\left(X_{0}^{2}+Y_{0}^{2}\right)-16\right], \\
& \mathcal{F}_{2}\left(X_{0}, Y_{0}\right)=-\frac{1}{64}\left[3 Y_{0}^{3}+\left(3 X_{0}^{2}-16\right) Y_{0}+32\right] .
\end{aligned}
$$

System $\mathcal{F}_{1}=\mathcal{F}_{2}=0$ has only one real solution

$$
\left(X_{0}^{*}, Y_{0}^{*}\right)=\left(0,-\frac{2}{3}\left(\frac{2 \cdot 2^{2 / 3}}{\sqrt[3]{9-\sqrt{65}}}+\sqrt[3]{2(9-\sqrt{65})}\right)\right)
$$

. Since the Jacobian

$$
\operatorname{det}\left(\left.\frac{\partial\left(\mathcal{F}_{1}, \mathcal{F}_{2}\right)}{\partial\left(X_{0}, Y_{0}\right)}\right|_{\left(X_{0}, Y_{0}\right)=(0,-2,98452)}\right)=0,167966 \ldots
$$

By Theorem 1 equation (19) has the periodic solution of the statement of the corollary.

Proof of Corollary 4. We have the equation

$$
u^{\prime \prime \prime \prime}+3 u^{\prime \prime}-4 u=\varepsilon\left(1-u^{2}\right) \sin 2 t,
$$

which corresponds to the case $p=-4, q=3$ and $F(t, x, y, z, v)=\left(1-x^{2}\right) \sin 2 t$. We obtain $\alpha=2$ and $\beta=1$. The functions $\mathcal{F}_{1}$ and $\mathcal{F}_{2}$ of Theorem 1 are

$$
\begin{aligned}
& \mathcal{F}_{1}\left(X_{0}, Y_{0}\right)=-\frac{1}{400} X_{0} Y_{0}, \\
& \mathcal{F}_{2}\left(X_{0}, Y_{0}\right)=\frac{3}{800} X_{0}^{2}+\frac{1}{800} Y_{0}^{2}-\frac{1}{2} .
\end{aligned}
$$

System $\mathcal{F}_{1}=\mathcal{F}_{2}=0$ has the four solutions $\left(X_{0}^{*}, Y_{0}^{*}\right)$ given by

Since the jacobian

$$
(0,20),(0,-20),\left(\frac{20}{\sqrt{3}}, 0\right),\left(-\frac{20}{\sqrt{3}}, 0\right) .
$$

$$
\operatorname{det}\left(\left.\frac{\partial\left(\mathcal{F}_{1}, \mathcal{F}_{2}\right)}{\partial\left(X_{0}, Y_{0}\right)}\right|_{\left(X_{0}, Y_{0}\right)=\left(X_{0}^{*}, Y_{0}^{*}\right)}\right)
$$

for these four solutions $\left(X_{0}^{*}, Y_{0}^{*}\right)$ is $-\frac{1}{400},-\frac{1}{400}, \frac{1}{400}, \frac{1}{400}$, respectively we obtain 
using Theorem 1 the four solutions given in the statement of the corollary.

Proof of Corollary 5. We have the equation

$$
u^{\prime \prime \prime \prime}+\frac{1}{36} u^{\prime \prime}+\frac{13}{36} u=\varepsilon(1-u) \sin t
$$

which corresponds to the case $p=\frac{1}{36}, q=\frac{13}{36}$ and $F(t, x, y, z, v)=(1-x) \sin t$. We obtain $m=1, n=2, r=1, s=3$. The functions $\mathcal{F}_{k}=\mathcal{F}_{k}\left(X_{0}, Y_{0}, Z_{0}, V_{0}\right)$ for $k=1,2,3,4$ of Theorem 2 are

$$
\begin{aligned}
& \mathcal{F}_{1}=-\frac{8}{1925}\left(10395 \pi X_{0}-33534 \sqrt{3} Z_{0}-33534 V_{0}+3850\right), \\
& \mathcal{F}_{2}=-\frac{216}{1925}\left(385 \pi Y_{0}+648 Z_{0}-648 \sqrt{3} V_{0}\right), \\
& \mathcal{F}_{3}=-\frac{81}{15400}\left(-9216 \sqrt{3} X_{0}-17664 Y_{0}+11088 \sqrt{3} Z_{0}+33264 V_{0}-1925\right), \\
& \mathcal{F}_{4}=\frac{27}{15400}\left(27648 X_{0}-52992 \sqrt{3} Y_{0}-99792 Z_{0}+33264 \sqrt{3} V_{0}+1925 \sqrt{3}\right) .
\end{aligned}
$$

System $\mathcal{F}_{1}=\mathcal{F}_{2}=\mathcal{F}_{3}=\mathcal{F}_{4}=0$ has only one real solution $\left(X_{0}^{*}, 0, Z_{0}^{*}, V_{0}^{*}\right)$ where $X_{0}^{*}, Z_{0}^{*}$ and $V_{0}^{*}$ are given in the statement of the corollary. Since the Jacobian

$$
\operatorname{det}\left(\left.\frac{\partial\left(\mathcal{F}_{1}, \mathcal{F}_{2}, \mathcal{F}_{3}, \mathcal{F}_{4}\right)}{\partial\left(X_{0}, Y_{0}, Z_{0}, V_{0}\right)}\right|_{\left(X_{0}, Y_{0}, Z_{0}, V_{0}\right)=\left(X_{0}^{*}, 0, Z_{0}^{*}, V_{0}^{*}\right)}\right)=0,0779343 \ldots,
$$

by Theorem 2 equation (20) has the periodic solution of the statement of the corollary.

\section{ACKNOWLEDGEMENTS}

The first author is partially supported by a MEC/FEDER grant MTM200803437, by a CIRIT grant number 2009SGR-410 and by ICREA Academia.

\section{REFERENCES}

[1] G.J.B. van den Berg, L.A. Peletier and W.C. Troy, Global branches of multi-bump periodic solutions of the Swift-Hohenberg equation, Arch. Ration. Mech. Anal. 158 (2001), 91- 153.

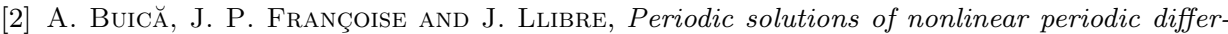
ential systems with a small parameter, Comm. on Pure and Appl. Anal. 6 (2007), 103-111.

[3] A.V. Buryak And N.N. AKhmediev, Stability criterion for stationary bound states of solitons with radiationless oscillating tails, Phys. Rev. E 51 (1995), 3572-3578.

[4] P.C. Carrião, L.F.O. Faria and O:H. Miyagaki, Periodic solutions for extended FisherKolmogorov and Swift-Hohenberg equations by truncature techniques, Nonlinear Anal. 67 (2007), 3076-3083.

[5] A.R. Champneys, Homoclinic orbits in reversible systems and their applications in mechanics, fluids and optics, Physica D 112 (1998), 158-186.

[6] J. GuCKenheimer and P. Holmes, Nonlinear oscillations, dynamical systems and bifurcations of vectors fields, Springer, 1983.

[7] M. KARLSSON AND A. HöÖK, Soliton-like pulses governed by fourth order dispersion in optical fibres, Opt. Com. 104 (1994), 303-307.

[8] J. LlibRe AND A.MAKhlouf, On some periodic orbits of the fourth-order differential equation $u^{\prime \prime \prime \prime}+q u^{\prime \prime}+p u=\varepsilon F\left(u, u^{\prime}, u^{\prime \prime}, u^{\prime \prime \prime}\right)$, submitted.

[9] J. Llibre, A.MAKhlOUf AND S. BADI, 3-dimensional Hopf bifurcation via averaging theory of second order, Dis. Cont. Dyn. Sys. 25 (2009), 1287-1295. 
[10] J. Llibre, S. Rebollo-Perdomo and J. Torregrosa, Limit cycles bifurcating from isochronous surfaces in $\mathbb{R}^{3}$, Advances Nonlinear Studies 11 (2011), 377-389.

[11] J. Llibre And M.A. TeixeIra, On the periodic orbits of a fourth-order differential equation, to appear in J. Math. Anal. and Appl., 2011.

[12] J. Llibre AND C. VAlls, Hopf bifurcation for some analytic differential systems in $\mathbb{R}^{3}$ via averaging theory Dis. Cont. Dyn. Sys. 30 (2011), 779-790.

[13] I.G. MaLkin, Some problems of the theory of nonlinear oscillations, (Russian) Gosudarstv. Izdat. Tehn.-Teor. Lit., Moscow, 1956.

[14] L.A. Peletier And W.C. Troy, Spatial Patterns, Higher Order Models in Physics and Mechanics, Brikhäuser, 2001

[15] M. Roseau, Vibrations non linéaires et théorie de la stabilité, (French) Springer Tracts in Natural Philosophy, Vol.8 Springer-Verlag, Berlin-New York, 1966.

[16] J. Sanders and F. Vehrulst, Averaging method in nonlinear dynamical systems, Applied Mathematical Sciences. 59, Springer, 1985.

[17] F. Vehrulst, Nonlinear differential equations and dynamical systems, Universitext, Springer 1996.

1 Departament de Matematiques, Universitat Autònoma de Barcelona, 08193 Bellaterra, Barcelona, Catalonia, Spain

E-mail address: jllibre@mat.uab.cat

2 Department of Mathematics, University of Annaba, Elhadjar, 23 Annaba, Algeria

E-mail address: makhloufamar@yahoo.fr 